\title{
Warum die Hemmung von IL-23 bei ankylosierender Spondylitis nicht wirksam war
}

\author{
Dennis McGonagle ${ }^{a, b} \quad$ Abdulla Watad ${ }^{a, c, d}$ Kassem Sharifa, c, d Charlie Bridgewood ${ }^{a}$ \\ ${ }^{a}$ Leeds Institute of Rheumatic and Musculoskeletal Medicine, University of Leeds, Leeds, Vereinigtes Königreich; ${ }^{b}$ National Institute \\ for Health Research (NIHR), Leeds Biomedical Research Centre (BRC), Leeds Teaching Hospitals, Leeds, Vereinigtes Königreich; \\ 'Department of Medicine 'B', Zabludowicz Center for Autoimmune Diseases, Sheba Medical Center, Tel-Hashomer, Israel; \\ ${ }^{\mathrm{d}}$ Sackler Faculty of Medicine, Tel-Aviv University, Tel-Aviv, Israel
}

\section{Schlüsselwörter}

IL-23 · Psoriasis-Arthritis · Ankylosierende Spondylitis · Enthese · $\mathrm{IL}-17$

\section{Zusammenfassung}

Der Begriff Spondyloarthritis (SpA) bezieht sich sowohl auf die axiale als auch auf die periphere Arthritis und schließt die ankylosierende Spondylitis (AS) und die Psoriasis-Arthritis (PsA) ein. Letztere ist eng mit der Psoriasis und der im Zusammenhang mit chronisch entzündlichen Darmerkrankungen (CED) auftretenden Arthritis assoziiert. Die Begründung für die Bedeutung von Interleukin-23 (IL23) bei Erkrankungen aus dem Formenkreis der Spondyloarthritiden stützt sich auf vier Quellen: Erstens wurde in genomweiten Assoziationsstudien (GWAS) nachgewiesen, dass alle genannten Erkrankungen Einzelnukleotid-Polymorphismen (SNPs) des IL23-Rezeptor (IL-23R)-Signalwegs aufweisen, wohingegen HLA-B27 nicht mit diesen Erkrankungen in Zusammenhang steht, was bedeutet, dass der IL-23-Signalweg der gemeinsame genetische Nenner ist. Zweitens zeigten tierexperimentelle Modelle, dass die IL-23/ IL-17-Achse eine Schlüsselrolle bei der SpA-bezogenen Arthropathie spielt, die sich initial in Form einer Enthesitis, aber auch als Synovitis und axiale Entzündung sowie als assoziierte Inflammation der Aortenwurzel und der Haut manifestiert. Drittens stützen auch die neu aufkommenden Erkenntnisse zur Immunologie der menschlichen Enthese das Vorliegen von IL-23-bildenden myeloischen Zellen nicht nur an der Enthese, sondern auch an anderen SpA-assoziierten Lokalisationen, einschließlich Haut und Darm. Und schließlich zeigen Arzneimittel, die auf den IL-23-Signalweg ausgerichtet sind, eine ausgezeichnete Wirksamkeit bei Hauterkrankungen und Wirkung bei CED und bei der mit SpA assoziierten peripheren Arthropathie. Die Tatsache, dass die IL-23-Blockade bei
AS, bei der es sich im Endeffekt um eine spinale Polyenthesitis handelt, augenscheinlich versagt, jedoch Belege für die Wirksamkeit der IL-23-Hemmung bei peripherer Enthesitis bei PsA und erste Hinweise auf einen Nutzen bei axialer PsA vorliegen, wirft viele Fragen auf. Die entscheidende Frage ist, ob es bei der spinalen Entzündung unter Umständen zu einer von IL-23 unabhängigen enthesialen IL-17A-Bildung kommt, wohingegen die periphere Enthesitis hauptsächlich von der IL-23-gesteuerten IL-17-Bildung abhängt. Ferner können Strategien zur IL-23-Blockade in Tiermodellen zwar die Entwicklung einer experimentellen SpA verhindern, nicht jedoch eine bestehende Erkrankung. Dies spricht dafür, dass IL-23 möglicherweise eine Rolle bei der Auslösung von Störungen der angeborenen Immunität spielt, während eine chronische Erkrankung von Reaktionen der T-Gedächtniszellen abhängt, die die IL-17A-Bildung unabhängig von IL-23 beeinflussen. Doch sind diesbezüglich noch weitere Untersuchungen erforderlich. Außerdem ist die IL-12/23-Dosierung bei entzündlichen Darmerkrankungen wesentlich höher als die in Studien zur AS verwendete Dosierung, was ebenfalls zu berücksichtigen ist. Aus den genannten Gründen spielt der IL-23-Signalweg im Konzept der SpA eine zentrale Rolle, doch müssen die Nuancen und Feinheiten bei der Entzündung des Achsenskeletts, die auf ein Nicht-Ansprechen auf den IL-23-Antagonismus hindeuten, noch formal definiert werden. Da keine vergleichenden immunologischen Untersuchungen der verschiedenen Skelettlokalisationen vorliegen, sind die Erklärungen zum jetzigen Zeitpunkt rein hypothetisch.

(c) 2021 McGonagle, Watad, Sharif and Bridgewood 


\section{Einleitung}

Die bahnbrechenden klinischen Beobachtungen von Moll und Wright [1] in den 1970er-Jahren führten zur Klassifizierung verschiedener Erkrankungen unter dem Oberbegriff Spondyloarthropathie (SpA) auf der Grundlage gemeinsamer klinischer und immunologischer Merkmale. Zu diesen Erkrankungen gehörten die ankylosierende Spondylitis (AS), die Psoriasis-Arthritis (PsA) (in Erweiterung des Psoriasis-Spektrums), die mit chronisch entzündlichen Darmerkrankungen (CED) assoziierte Arthropathie einschließlich Morbus Crohn und Colitis ulcerosa, die enterogene und urethrogene reaktive Arthritis sowie die anteriore Uveitis, die ebenfalls mit diesen Erkrankungen assoziiert ist $[1,2]$. Eine Gemeinsamkeit all dieser Erkrankungen war eine Entzündung des Achsenskeletts, eine periphere Oligoarthritis der unteren Extremitäten, in einigen Fällen eine Enthesitis, ein Zusammenhang mit einer Infektion oder Funktionsstörung des Darms sowie Rheumafaktor-Negativität $[4,5]$. Ursprünglich enthielt das Konzept keinen Vorschlag für ein einheitliches pathologisches Verständnis der SpA-assoziierten Arthropathie.

Kurz nach dem von Moll und Wright entwickelten SpA-Konzept wurde HLA-B27 (humanes Leukozytenantigen B27) entdeckt, das mit AS, PsA mit Beteiligung des Achsenskeletts, CED-bezogener axialer Arthritis, anteriorer Uveitis und reaktiver Arthritis assoziiert war [6-8]. Die CED selbst oder die CED-bezogene periphere Arthropathie waren jedoch nicht mit HLA-B27 assoziiert. Aufgrund der klinischen Merkmale des Morbus Behçet (Bechet's disease, BD) schlugen die Forscher in einer seit 4 Jahrzehnten viel zitierten Arbeit vor, auch diese Krankheit dem SpA-Konzept zuzuordnen [9]. Wegen der fehlenden Sakroiliitis und der fehlenden starken Assoziation mit HLA-B27 wurde die BD nie auf breiter Front in das vorgeschlagene Klassifikationsschema aufgenommen. Wie in der folgenden Diskussion erläutert, zeigten GWASStudien jedoch, dass die mit dem Interleukin (IL)-23-Signalweg verbundenen genetischen Polymorphismen über das gesamte Spektrum der SpA-Arthropathie, einschließlich AS und PsA, sowie bei Psoriasis und CED und auch bei BD auftreten, und bestätigten damit auf ganzer Linie das von Moll und Wright beschriebene Konzept $[9,10]$.

\section{Aktuelle Therapie bei AS}

$\mathrm{Zu}$ den derzeitigen Therapieoptionen bei AS gehören Anti-Tumornekrosefaktor (TNF)-Wirkstoffe zur Behandlung von Patienten, die nicht auf nichtsteroidale Antiphlogistika (non-steroidal anti-inflammatory drugs, NSAIDs) ansprechen. Wenn AntiTNF-Wirkstoffe kontraindiziert sind oder ihre Wirksamkeit nachlässt, kann eine Behandlung mit einem von zwei AntiIL-17A-Blockern erwogen werden, unter dem Vorbehalt, dass diese Wirkstoffe nicht bei einer aktiven assoziierten CED eingesetzt werden dürfen [11]. In den kommenden Jahren werden wahrscheinlich JAK-Inhibitoren in der klinischen Behandlung der AS zur Anwendung kommen [12]. Zwar weisen Guselkumab und Ustekinumab eine gewisse Wirksamkeit bei der PsA-assoziierten
Erkrankung des Achsenskeletts auf [13, 14], doch haben Studien mit Ustekinumab und Risankizumab, dem anderen p19-Blocker, keine Belege für eine Wirksamkeit dieser Wirkstoffklasse bei AS erbracht $[15,16]$.

\section{Die IL-23/IL-17-Achse}

Naive T-Zellen sind in der Lage, sich in Abhängigkeit von der lokalen Mikroumgebung in Effektor-T-Zellen zu differenzieren, wenn sie in lymphatischem Gewebe auf ein verwandtes Antigen treffen. Dies beinhaltet die Peptid-Präsentation durch den HauptHistokompatibilitätskomplex (major histocompatibility complex, MHC) gegenüber dem T-Zell-Rezeptor (T cell receptor, TCR) (Signal 1) und die anschließende Koaktivierung mit Bindung von CD80/86 an CD28 (Signal 2) auf den T-Zellen [17]. Zytokinstimuli wie IL-1 $\beta$, IL-6, IL-21 und/oder IL-23 können beim Menschen zur Bildung von IL-17 durch T-Zellen führen, wobei CD4-positive Th17-Zellen und CD8-positive Tc17-Zellen die am besten beschriebenen sind [18]. Diese IL-23-aktivierten T-Zellen sezernieren zudem eine Reihe von anderen Zytokinen wie IL-17F, IL-22 und TNF [18].

\section{Die genetische Begründung des IL-23-Signalwegs bei Erkrankungen aus dem SpA-Spektrum}

Interessanterweise wurden IL-23-Rezeptor (IL-23R)-Polymorphismen bei allen oben genannten Krankheitsklassen berichtet, nicht jedoch bei den klassischen Autoimmunerkrankungen (Abb. 1). Darüber hinaus treten Berichten zufolge bei allen der genannten Krankheiten verschiedene Einzelnukleotid-Polymorphismen (single-nucleotide polymorphisms, SNPs) des IL-23-Signalwegs auf, darunter solche, die das Zytokin IL-23 selbst betreffen, und solche, die die nachgeschalteten JAK2- und Tyk2-, STAT3- und IL-17RA-Signalwege betreffen $[5,9,19]$. Eine Vielzahl weiterer Daten zu genetischen Polymorphismen hat diese Befunde insoweit bestätigt, als dass sie zeigten, dass die klassischen Autoimmunerkrankungen eine vollkommen andere, nicht mit dem IL-23-Signalweg in Zusammenhang stehende genetische Architektur aufweisen [20]. Die SNPs im IL-23R-Signalweg sind zudem mit CED [21] und BD assoziiert [22], was die von Moll und Wright und Kollegen vorgeschlagene historische Verbindung mit SpA stützt. Der SNP im IL-23R (R381Q) wirkt protektiv gegen CED, AS und Psoriasis [23-25]. Auf funktioneller Ebene führt er zu einem Funktionsverlust und einer geringeren STAT3-Aktivierung und damit zu einer verminderten IL-17-Bildung durch T-Zellen $[26,27]$. Daher scheint eine «vollkommen normale» IL-23-Signalübermittlung und -Funktion, die höher ist als bei Probanden mit dem R381Q-Polymorphismus, mit AS in Zusammenhang zu stehen. Es ließe sich die Hypothese aufstellen, dass eine Anti-IL23-Therapie zu einer weiteren Verringerung und Angleichung an das mit dem protektiven Allel assoziierte Ausmaß der Bildung führen würde. Studien zu AS haben dies jedoch nicht bestätigt. Zwar ist der IL-23-Signalweg genetisch in allen oben genannten Geweben beteiligt, doch ist der Unterschied im relativen Beitrag 


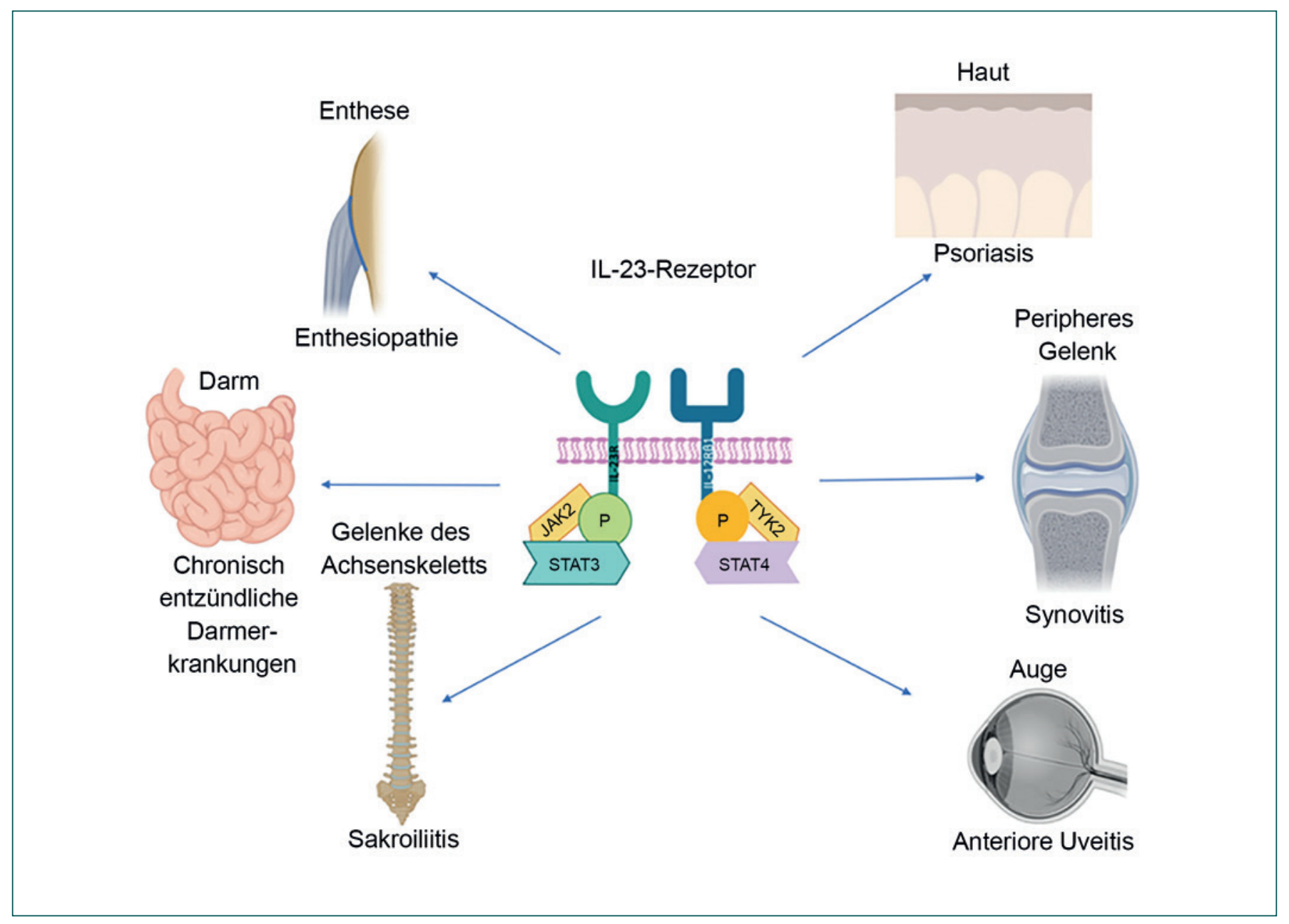

Abb. 1. Mit IL-23R-Polymorphismus assoziierte Krankheiten. Der IL-23-Zytokinweg ist auf genetischer und zellulärer immunologischer Ebene eng mit okulären, kutanen und intestinalen Erkrankungen und Arthropathien verknüpft. Bei den unterschiedlichen Organreaktionen besteht eine starke immunologische Heterogenität. So wirkt beispielsweise das TNF-Fc-Fusionsprotein Etanercept nicht bei CED und ist bei anteriorer Uveitis generell nicht wirksam. Die Bedeutung von IL-23-Blockern bei anteriorer Uveitis muss noch näher bestimmt werden, doch geht man allgemein davon aus, dass Anti-IL-17A-Blocker bei dieser SpA-Domäne nicht wirksam sind. Alle Wirkstoffe mit antagonistischer Wirkung auf die IL-23/17-Achse weisen eine hohe Wirksamkeit bei Psoriasis im Vergleich zu Gelenkerkrankungen auf. Bei den Darmerkrankungen spielen die IL-17-Blocker eine wichtige Rolle in Hinblick auf die Barrierefunktion an dieser Körperstelle, was eine Erklärung für das Auftreten von CED-Schüben unter Anti-IL-17A-Therapie sein könnte. Möglicherweise sind die 3 Zytokine TNF, IL-17A und IL-23 alle gleichermaBen bedeutsam für die periphere Arthritis bei PsA und auch für die Enthesitis im peripheren Skelett, denn unter therapeutischem Antagonismus ist ein gutes Ansprechen zu beobachten. Der gegen IL-12 und IL-23 gerichtete Anti-p40-Wirkstoff ist unter Umständen bei PsA weniger wirksam als die anderen 3 Kategorien von Medikamenten; allerdings sind diesbezüglich weitere Studien erforderlich. Und schließlich zeigten nur TNF- und IL-17-Blocker eine Wirksamkeit im Achsenskelett, wohingegen die IL-23-Blockade mittels p40- oder p19-Blockern nicht funktionierte. In den vergangenen Jahren ist die lokalisationsspezifische Kompartimentierung der Immunität ins Zentrum der Aufmerksamkeit gerückt und diese spiegelt vermutlich gewebespezifische Faktoren und Wechselwirkungen mit der Mikrobiota wider, die unterschiedliche Immunantworten zur Folge haben.

von IL-23 und anderen Zytokinen bei den verschiedenen SpA-assoziierten Erkrankungen mit einer unterschiedliche Wirksamkeit verbunden, wie klinische Studien belegen (Abb. 2).

\section{Gewebemikroanatomie des IL-23-Signalwegs und Tier- modelle}

Es ist allgemein bekannt, dass die Entzündung bei rheumatoider Arthritis (RA) primär das Synovialgewebe angreift und mit einer Autoimmunität gegen citrullinierte Synovialproteine verbunden ist. Diese löst eine Entzündungsreaktion aus, die eine periartiku- läre Gelenkzerstörung und -erosion zur Folge hat, wobei der Phänotyp der polyartikulären Gelenkzerstörung gut bekannt ist. Mitte der 1990er-Jahre durchgeführte Magnetresonanztomografie (MRT)-Untersuchungen zeigten, dass die Enthesitis in geschwollenen kleinen wie großen Gelenken bei PsA und SpA im Allgemeinen nachweisbar war [28]. Dies führte zu dem Enthesitis-basierten Krankheitsmodell der SpA, bei dem angenommen wurde, dass zytokinvermittelte primäre Entzündungsreaktionen an der Enthese eine angrenzende Synovitis, Tenosynovitis und Osteitis verursachen [29-31]. Im weiteren Verlauf wurde in Tiermodellen nachgewiesen, dass eine Fehlregulation der TNF-Bil- 


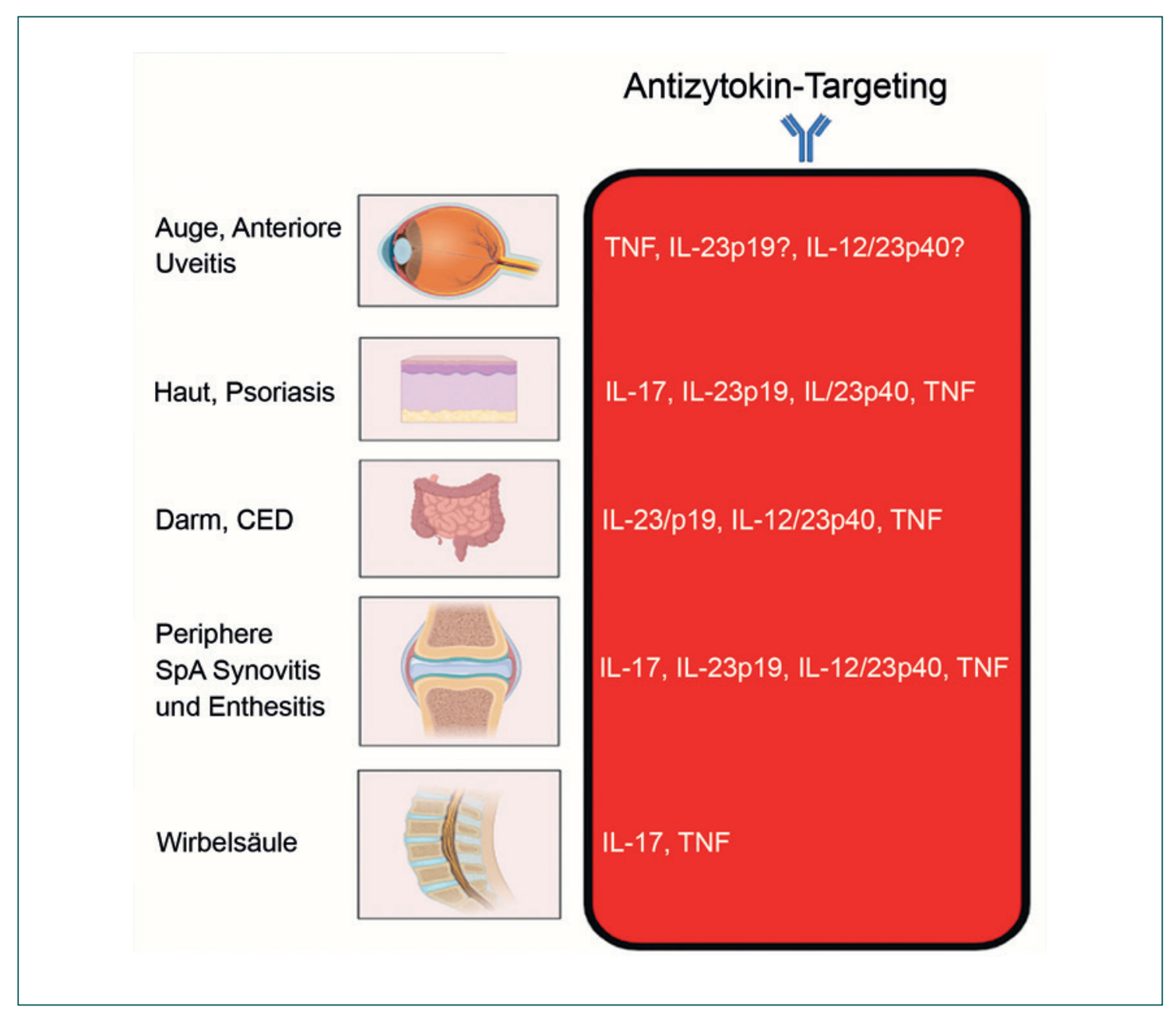

Abb. 2. Wirksamkeit der Zytokinblockade in verschiedenen Organen. Die klassischen MHC-Klasse-II-assoziierten Autoimmunerkrankungen, die durch die Bildung von Autoantikörpern gekennzeichnet sind, segregieren in Familien und Individuen und betreffen überwiegend Frauen. Die Gruppe der SpA-Erkrankungen zeigt keine wesentlichen Unterschiede hinsichtlich der Geschlechterverteilung; sie weist genetische Assoziationen mit der MHC-Klasse 1 auf, spezifische, bestätigte mit der Krankheit assoziierte Autoantikörper fehlen und Manifestationsort der Krankheit sind verletzte oder physisch belastete Körperstellen. Genetische Untersuchungen und die experimentelle Immunologie haben eindeutig bestätigt, dass der IL-23-Signalweg in dieser Mischung eine zentrale Rolle spielt. Da IL-23 sowohl IL-22 als auch IL-17 reguliert, sind wir der Auffassung, dass der IL-23-Signalweg neben der Immunität, einschließlich der antimykotischen Immunität über die IL-17-Regulation, eine Feinabstimmung der Gewebereparatur an Körperstellen mit Verletzungen und physischem oder chemischem Stress, wie zum Beispiel im Darm, bewirkt. Das TNF-Fc-Fusionsprotein Etanercept wirkt nicht bei CED und ist bei anteriorer Uveitis generell nicht wirksam.

dung an der Enthese eine polyartikuläre Gelenkzerstörung verursacht, was die Enthesitis als Krankheitsmechanismus zusätzlich bestätigte [32, 33].

In einer grundlegenden Arbeit wiesen Sherlock et al. [34] eine IL-23R-exprimierende Zellpopulation in der normalen Enthese von Mäusen nach. Später wurde bestätigt, dass dieses Modell Tyk2-abhängig ist (Tyk2 vermittelt die IL-23-Signalübermittlung) [35], während in derselben Arbeit festgestellt wurde, dass Tyk2SNPs beim Menschen mit einem Fortschreiten der AS korrelieren. Im IL-23-Minicircle-Modell führte die distale Überexpression des IL-23-Zytokins in der murinen Leber mittels DNA-MinicircleTechnologie schnell zu einer peripheren Enthesitis, die sich auf das Synovialgewebe und den Knochen ausbreitete und eine polyartikuläre Gelenkzerstörung zur Folge hatte. Im Modell der IL-23-ab- hängigen Enthesitis von Sherlock et al. [34] wurde später von Reinhardt et al. [36] gezeigt, dass es sich bei den meisten IL-17A-bildenden Zellen in der normalen murinen Enthese um IL-23Rexprimierende $\gamma \delta$-T-Zellen handelte. Diese Zellpopulation ist heterogen und hat unterschiedliche Funktionen wie beispielsweise frühe angeborene Immunantworten und das Priming der adaptiven Immunität und spielt eine wichtige Rolle bei der Reparatur des Gewebes [37]. Die Bedeutung von IL-23 im SpA-Konzept wurde in diesem Modell durch die Induktion einer psoriasiformen Hautentzündung, einer Inflammation der Aortenwurzel und durch die Entwicklung einer axialen Entzündung stark gefestigt [34]. Andere Untersucher, die die gleiche Minicircle-Technologie verwendeten, hoben die Bedeutung der schweren Synovitis und Knochenerosion sowie von RA-ähnlichen Merkmalen hervor [38]. 
Natürlich existieren verschiedene unabhängige Tiermodelle, in denen die Schlüsselrolle von IL-23 bei experimentellen Darmentzündungen und Arthritis vom reaktiven Typ nachgewiesen wurde $[39,40]$. Es liegen zunehmend Hinweise vor, nach denen die intestinale Mikrobiota zudem mit der Interdependenz und Kreuzregulation der IL-23/17-Achse in Verbindung steht, und dieser Bereich ist derzeit Gegenstand aktiver Forschung [41-44]. Ein weiteres Beispiel für eine IL-23-abhängige SpA liefert das SKG-Mausmodell, das zahlreiche SpA-Merkmale in einem IL-23/17-Achsen-abhängigen Signalweg aufweist [45]. Die SKGMaus trägt eine Punktmutation im ZAP-70-Gen, die zu einer verringerten TCR-Signalübermittlung führt und die Entwicklung einer entzündlichen Multiorganerkrankung und SpA-ähnlichen Erkrankung nach Gabe von fungalen oder bakteriellen Adjuvanzien zur Folge hat [45]. Insgesamt stützen diese Modelle die Vorstellung, dass eine topografisch auf die Enthese zentrierte Entzündung die Krankheit fördert [31].

\section{Neu aufkommende immunologische Erkenntnisse zum IL-23-Signalweg und der IL-23/17-Achse bei SpA beim Menschen}

Klinische Studien mit Menschen analysieren die Immunpathogenese der Krankheit beim Menschen, und es ist wichtig, das Augenmerk darauf zu richten, um die humane Enthesitis besser zu verstehen. Erstens haben sich IL-17A-Blocker sowohl bei der peripheren als auch bei der axialen SpA als wirksam erwiesen, und dies beinhaltete auch den Wirksamkeitsnachweis bei isolierter Enthesitis als sekundäres Zielkriterium [46-50]. Ebenso wurde in der veröffentlichten Literatur die Wirksamkeit von IL-12/IL23-p40-Blockern bei peripherer PsA und isolierter Enthesitis nachgewiesen [30, 51-53]. Neuere Studien haben außerdem gezeigt, dass eine IL-23-p19-Blockade bei peripherer Synovitis und der verwandten Enthesitis wirksam ist [54, 55]. Diese Ergebnisse für sich genommen deuten auf eine biologische Rolle von IL-23 bei der nichtaxialen peripheren Enthese hin [56], doch was ist die biologische Grundlage hierfür?

In Anknüpfung an die Studie von Sherlock et al. [34] untersuchte unsere Gruppe das Vorliegen von Zytokinen der IL-23/17-Achse in der normalen spinalen Enthese beim Menschen. Wir identifizierten ortsständige angeborene lymphoide Zellen der Gruppe 3 in normalem spinalen Enthese-Knochen- und Weichgewebe, die IL-23R exprimierten [57]. Die IL-23/IL-1 $\beta$-Stimulation von normalem menschlichem Enthese-Gewebe hatte eine Hochregulation des IL-17A- und IL-17F-Transkripts zur Folge [57]. Darüber hinaus hatten wir zuvor über das Vorhandensein von Makrophagen bei akuter Enthesitis beim Menschen berichtet [58]. Dies warf die Möglichkeit auf, dass in der menschlichen Enthese unter Umständen eine lokale IL-23-Produktion erfolgt, und im weiteren Verlauf wurde gezeigt, dass die normale Enthese eine IL-23-induzierbare Proteinproduktion aus CD14-positiven myeloischen Zellen nach bakterieller oder fungaler Stimulation aufweist [59]. Zudem stellten wir fest, dass sich diese IL-23-Sekretion durch die Gabe von Phosphodiesterase-4 (PDE4)-Blockern abschwächen ließ, was translational bedeutsam sein könnte, da die Antagonisierung dieses Signalwegs beim Menschen Wirksamkeit bei peripherer Enthesitis bei PsA zeigt [56]. Sowohl TNF als auch IL-17A sind in der Lage, die Osteogenese in vitro in mesenchymalen Stammzellen (mesenchymal stem cells, MSCs) aus der spinalen Enthese zu induzieren $[60,61]$.

\section{Komplexität des IL-23-Signalwegs in der Wirbelsäule und andere Merkmale der SpA}

Seitdem die IL-23-Blockade bei der AS gescheitert ist, gibt es umfangreiche wissenschaftliche Spekulationen darüber, was der Grund dafür sein könnte [62]. Interessanterweise sind die Krankheiten der Gruppe der SpA-Erkrankungen zwar eng miteinander verbunden, unterscheiden sich jedoch gleichzeitig in ihrer Immunpathologie zwischen den verschiedenen Lokalisationen, was sich am besten in der Nicht-Wirksamkeit von Therapien bei einigen Krankheitsdomänen zeigt (Tabelle 1). So ist beispielsweise das TNF-Fusionsprotein Etanercept am Skelett wirksam, jedoch nicht im Darm [68]. Ebenso besitzen IL-17A-Blocker eine eindrucksvolle Wirksamkeit in der Haut und eine gute Wirksamkeit im Skelett, wohingegen sie im Darm unwirksam sind und unter bestimmten Umständen mit CED-Schüben in Verbindung stehen [69]. Laboruntersuchungen im Anschluss an die fehlgeschlagenen Humanstudien mit Anti-IL-17A bei Morbus Crohn ergaben, dass die IL-17A-Bildung durch $\gamma \delta$-T-Zellen im Darm unabhängig von der IL-23R-Signalübermittlung erfolgt, wo die IL-17-Signalübermittlung für die Aufrechterhaltung der intestinalen OkkludinJunctions erforderlich ist [70].

Die oben beschriebene Wirksamkeit der IL-17-Blockade bei axialer Erkrankung und die fehlende Wirksamkeit der IL-17A-Hemmung im Darm werfen die Frage nach einer möglichen IL-17-Bildung in der Wirbelsäule auf, die unabhängig von IL-23 erfolgt und die für die eigenartige berichtete fehlende Wirksamkeit der IL-23-Signalweghemmung bei axialer Erkrankung verantwortlich sein könnte. In 2 Studien zur Blockade des IL-23-Signalwegs, einschließlich der p40- und p19-Blockade, zeigte diese keine Wirksamkeit bei AS, allerdings waren unter p40-Antagonismus marginale, statistisch nicht signifikante Verbesserungen der CRP-Werte (C-reaktives Protein) und leichte Verbesserungen im MRT zu beobachten [15, 16]. 2 Phase-II-Studien zeigten die Wirksamkeit von p19-Blockern bei der peripheren Arthropathie der PsA einschließlich peripherer Enthesitis [54, 55, 71]. Dies hat ein neues Rätsel aufgeworfen: Wie kann es sein, dass ein Medikament bei der peripheren skelettalen Enthesitis wirkt, nicht jedoch bei der axialen Enthesitis, die den überwiegenden Teil der AS-Pathologie außerhalb des Iliosakralgelenks ausmacht? Ein wichtiger Unterschied könnte darin bestehen, dass im peripheren Skelett synovial-enthesiale Komplexe vorkommen, in der Wirbelsäule dagegen nicht [72].
104

Kompass Autoimmun 2021;3:100-107 DOI: 10.1159/000518361 
Tab. 1. Heterogenität der Erkrankungen aus dem Spondylarthropathie-Spektrum in Hinblick auf das Ansprechen auf die Immuntherapie

\begin{tabular}{|c|c|c|c|c|c|}
\hline Signalweg & Wirkstoff & Unerwünschte Ereignisse & Immunpathologie & Empfehlungen & Ref. \\
\hline TNF & $\begin{array}{l}\text { Infliximab, Etanercept, } \\
\text { Adalimumab, } \\
\text { Certolizumab Pegol, } \\
\text { Golimumab }\end{array}$ & $\begin{array}{l}\text { Periphere Arthralgie bei der } \\
\text { CED-Behandlung, paradoxe } \\
\text { Psoriasis }\end{array}$ & $\begin{array}{l}\text { Paradoxe Hochregulation der Interferon- } \\
\text { Signalwege }\end{array}$ & $\begin{array}{l}\text { Umstellung auf IL-23- } \\
\text { oder (außer bei CED) } \\
\text { IL-17-Inhibitoren }\end{array}$ & {$[63,64]$} \\
\hline TNF & (Etanercept) & $\begin{array}{l}\text { Uveitis, fehlende Wirksamkeit } \\
\text { bei CED }\end{array}$ & $\begin{array}{l}\text { Mechanismus unklar; im Darm möglicher } \\
\text { Zusammenhang mit Fact-Antikörpern; in } \\
\text { myeloischen Zellen möglicher } \\
\text { Zusammenhang mit antikörperabhängiger } \\
\text { Zytotoxizität }\end{array}$ & $\begin{array}{l}\text { Umstellung auf einen } \\
\text { anderen TNF-Blocker }\end{array}$ & {$[63,64]$} \\
\hline IL-17 & $\begin{array}{l}\text { Secukinumab, } \\
\text { Brodalumab, } \\
\text { Ixekizumab }\end{array}$ & CED & $\begin{array}{l}\text { Dysregulation der intestinalen epithelialen } \\
\text { Permeabilität, die durch IL-17A (Tight } \\
\text { Junctions) reguliert wird }\end{array}$ & $\begin{array}{l}\text { Umstellung auf TNF- } \\
\text { oder IL-23- } \\
\text { Inhibitoren }\end{array}$ & [65] \\
\hline $\begin{array}{l}\text { IL-23 (p40- und } \\
\text { p19-Blocker) }\end{array}$ & $\begin{array}{l}\text { Ustekinumab, } \\
\text { Rasinkizumab }\end{array}$ & $\begin{array}{l}\text { Fehlende Evidenz für die } \\
\text { Wirksamkeit bei AS }\end{array}$ & $\begin{array}{l}\text { Unklar, aber wahrscheinlich IL-17A-Bildung } \\
\text { unabhängig von IL-23 }\end{array}$ & & {$[15,16]$} \\
\hline a4 $\beta 7$-Integrin & Vedolizumab & Sakroiliitis und Synovitis & $\begin{array}{l}\text { abnormale intestinale Barrierefunktion und } \\
\text { Eintritt von bakteriellen Antigenen, Zytokinen, } \\
\text { Adjuvanzien und Molekülen mit } \\
\text { pathogenassoziiertem molekularen Muster in } \\
\text { den systemischen Kreislauf und Ablagerung } \\
\text { im peripheren Skelett in Regionen mit } \\
\text { enthesialem Gewebe }\end{array}$ & $\begin{array}{l}\text { Umstellung auf TNF- } \\
\text { oder IL-12/23-Blocker }\end{array}$ & {$[66,67]$} \\
\hline
\end{tabular}

\section{Neu auftretende zelluläre Akteure bei der Nichtlinearität zwischen IL-23- und IL-17-Signalwegen bei SpA}

Menschliche $\gamma \delta$-T-Zellen werden in die beiden Hauptgruppen $\delta 1$ und $\delta 2$ eingeteilt [73]. Wir haben das Konzept untersucht, nach dem beim Menschen eine Heterogenität dieser Populationen vorliegen könnte. Ortsständige $\gamma \delta$-T-Zell-Populationen finden sich sowohl im normalen enthesialen Weichgewebe der Wirbelsäule als auch im perienthesialen Knochen, wobei ihre Zahl im perienthesialen Knochen höher ist [74]. Wir stellten fest, dass bei der $\delta 1$-Population der ortsständigen enthesialen $\gamma \delta$-T-ZellPopulationen die IL-23R-Expression fehlte, wohingegen die $\delta 2$ Population diesen Rezeptor exprimierte. Nur bei der $\delta 2$ Population kam es zu einer Heraufregulation von IL-17 als Reaktion auf die IL-23-Signalübermittlung. Bei beiden Populationen konnte jedoch durch Stimulation mit Phorbol-12-myristat13-acetat (PMA) oder Anti-CD3/CD28 eine Expression von IL17A induziert werden [74]. Dies zeigt, dass die Komplexität des IL-23-Signalwegs auch die Wirbelsäule umfasst, und unsere Ergebnisse sprechen dafür, dass IL-17A, ein Schlüsselzytokin bei AS und spinaler Entzündung, möglicherweise nicht ausschließlich von IL-23 abhängt. Folglich handelt es sich bei der IL-23/IL-17AAchse um eine 2-seitige Medaille, bei der die IL-17A-Bildung unabhängig von IL-23 ist und sehr unterschiedliche biologische Auswirkungen auf die Entzündung des Darms und des Skeletts hat; während eine IL-17A-Blockade im ersten Fall schädlich ist, kann sie in letzterem potenziell von Nutzen sein $[75,76]$. In letzter Zeit sind andere Theorien zur IL-17-Sekretion unabhängig von IL-23 aufgekommen. Bei Mukosa-assoziierten invarianten T-Zellen (MAIT) handelt es sich um spezialisierte und den Zellen des angeborenen Immunsystems gleichende T-Zellen, die als Brücke zwischen angeborener und adaptiver Immunität fungieren. MAIT werden durch konservierte bakterielle Liganden aktiviert, die aus der Vitamin-B-Biosynthese stammen und die dem TCR vom MHC-Klasse-I-ähnlichen MR1 präsentiert werden [77]. Untersuchungen ergaben, dass MAIT nach TCR-Aktivierung und Stimulation durch IL-12 und IL-18 IL-17 bilden, das unabhängig von IL-23 ist [78]. Die menschliche Enthese enthält außerdem herkömmliche T-Zellen, und zwar sowohl CD4- als auch CD8-positive Zellen. Die enthesialen CD4- und CD8-positiven Zellen können nach TCR-Stimulation (Anti-CD3/CD28) IL-17A bilden, ohne dass eine zusätzliche IL-23-Stimulation erforderlich ist [79].

\section{IL-23-Blockade zur SpA-Prävention}

Diese neu aufkommenden Erkenntnisse zur Immunologie sind ursächlich auf die fehlgeschlagene Phase-II-Studie zu Risankizumab bei AS und die fehlgeschlagene Phase-III-Studie zu Ustekinumab bei AS zurückzuführen [80, 81]. Dies wurde in einem experimentellen SpA-Modell an HLA-B27/Huß2m-transgenen Ratten, die spontan SpA entwickeln, untersucht [82]. Die Tiere erhielten prophylaktisch Anti-IL-23R vor dem Auftreten der Krankheit oder Kontrollinjektionen. Dagegen wurde im anderen Fall bis zur vollständigen Manifestation der Krankheit abgewartet und anschließend erhielten die Tiere Anti-IL-23R-Antikörper oder die Kontrolle. Diese Untersuchungen zeigten, dass die IL23-Blockade die Entwicklung der Krankheit verhindern konnte, aber nicht in der Lage war, eine vollständig manifestierte Arthritis zu unterdrücken [82]. Wie sich dies genau auf den Menschen anwenden lässt, ist unklar, da die Feinheiten dieses Rattenmodells 
und seine Übertragbarkeit auf die menschliche SpA nicht vollständig geklärt sind [4]. So könnten die Ergebnisse beispielsweise auf eine Schlüsselrolle der Gedächtnis-T-Zellen hindeuten, die IL17A möglicherweise unabhängig von der IL-23-Signalübermittlung bilden. Allerdings wurde nie untermauert, dass CD8-positive T-Zellen bei der experimentellen SpA mit HLA-B27 eine Rolle spielen [83], wohingegen die Genetik der menschlichen SpA, einschließlich HLA-B27, ERAP-1 und verschiedener anderer SNPs, eher auf diesen Signalweg verweist [4].

Es liegen erste Hinweise vor, die diese Tiermodelle beim Menschen stützen. Kürzlich wurde gezeigt, dass die Blockade des IL23-Signalwegs mit Ustekinumab bei Psoriasis eine Rückbildung der subklinischen peripheren Enthesiopathie bewirkt [84]. Ob sich durch die Anwendung von IL-23-Blockern die Entwicklung einer axialen Entzündung bei Psoriasis-Patienten verhindern lässt, ist eine interessante und offene Frage. Erwähnenswert ist, dass eine Sekundäranalyse der zulassungsrelevanten Phase-IIIStudien zu Ustekinumab bei PsA eine Wirksamkeit bei axialer PsA einschließlich einer Besserung der Schmerzen im Wirbelsäulenbereich zeigte [13].

Als Zusammenfassung veröffentlichte neuere Studien haben gezeigt, dass bei Patienten mit PsA, die an Studien zur Polysynovitis teilnahmen und von denen jedoch $20 \%$ auch eine röntgenologisch nachgewiesene Sakroiliitis und Rückenschmerzen hatten, die p19-Blockade mit Guselkumab zu Verbesserungen der axialen Symptome führte [14]. Diese Studien deuten auf eine mögliche immunologische Heterogenität der entzündlichen Wirbelsäulenerkrankung hin, wobei einige Fälle von axialer Entzündung bei PsA eine direkte Rolle von IL-23 zeigen, die deutlicher und anders ausfällt als bei AS.

\section{Unklarheiten in Bezug auf IL-23 in der Wirbelsäule}

Es ist unwahrscheinlich, dass die p19-Blockade die Funktion des unzureichend charakterisierten Zytokins IL-39 stört, das ebenfalls die p19-Untereinheit (p19+EBI3) aufweist [85]. In der Tat handelt es sich bei diesem Zytokin beim Menschen nach wie vor um ein theoretisches Zytokin, für dessen Bildung oder Funktion in vivo keine Belege vorliegen $[85,86]$. Daher scheint es derzeit so zu sein, dass die p19-Blockade in erster Linie nur auf IL-23 und nicht auf ein anderes, noch schlecht charakterisiertes Zytokin wirkt, allerdings sind diesbezüglich weitere Untersuchungen erforderlich.

Die Wirbelsäulenentzündung bei AS tritt überwiegend im perienthesialen Knochen auf, und die Lokalisation der Erkrankung hängt mit dem genetischen HLA-B27-Status zusammen [58]. Unsere Untersuchungen an menschlichen Wirbelsäulen-Enthesen ergaben, dass die Bildung oder Induktion von IL-23 auf der Knochenseite der Enthese sehr viel höher ist [59]. Die Frage, ob sich dies in Therapeutika überführen lässt, ist noch offen und eventuell sind höhere Dosen von p19-Blockern erforderlich, um die axiale Entzündung zu lindern.

In der fehlgeschlagenen Studie zu Ustekinumab bei AS wurde ein Dosierungsschema mit 45 und $90 \mathrm{mg}$ verwendet, doch war die höhere Dosierung mit einer nichtsignifikanten CRP-Senkung und geringfügigen Verbesserungen der MRT-Läsionen verbunden [81]. Die bei Morbus Crohn verwendete Ustekinumab-Dosis beinhaltet eine intravenöse Aufsättigungsdosis von $6 \mathrm{mg} / \mathrm{kg}$ [87], was potenziell dem Schema einer 18-monatigen Behandlung mit Ustekinumab $90 \mathrm{mg}$ subkutan bei AS in der gescheiterten Studie entspricht. Für eine formale Beurteilung dieser Fragen besteht hier sicherlich Spielraum für Dosissteigerungen. Darüber hinaus wurde vermutet, dass p40-Blocker möglicherweise die immunregulatorischen Effekte von IL-12 in der Haut einschränken können [88], und hinsichtlich der möglichen negativen Auswirkungen der p40-Blocker außerhalb des IL-23-Signalwegs besteht ebenfalls Unsicherheit. Die negativen Ergebnisse der p19-Studie zur AS sprechen jedoch dagegen.

Es muss klar gesagt werden, dass das Verständnis dieser Signalwege durch die translationale Therapie beim Menschen und nicht durch experimentelle wissenschaftliche Untersuchungen im Labor geleitet wird. Interessant ist, dass p40-Blocker bei axialen Symptomen der PsA wirksam waren, doch ist zu berücksichtigen, dass es sich bei der HLA-B27-negativen axialen PsA um eine andere Erkrankung als AS handeln könnte [89]. Eine klinische Abkürzung zum Verständnis der Dosierungsprobleme im Zusammenhang mit IL-23-Blockern könnte aus der Untersuchung der Dosierung bei der Therapie von Morbus-Crohn-Patienten mit gleichzeitig bestehender axialer Erkrankung herrühren. Bedauerlicherweise existieren derzeit keine vergleichenden immunologischen Untersuchungen zwischen den spinalen und peripheren Enthesen, sodass es sich hierbei nach wie vor größtenteils um Mutmaßungen handelt.

\section{Schlussfolgerungen}

In der vorliegenden Arbeit wurde der Begriff SpA so gefasst, dass er die vielgestaltigen Manifestationsformen, die mit der axialen Entzündung assoziiert sind, darunter auch Haut- und Darmbeteiligung, einschließt, für die eindeutig nachgewiesen wurde, dass IL-23-SNPs einen gemeinsamen Nenner für die verschiedenen Erkrankungen bilden. Zudem hat sich in experimentellen und menschlichen Systemen klar gezeigt, dass die IL-23/IL-17-Achse an der Biologie von Haut, Darm und Enthesen beteiligt ist [90]. Innerhalb dieser Krankheitsdomänen gibt es Unterschiede in der Immunpathologie, die die kontextabhängige Biologie der verschiedenen Gewebe widerspiegeln. Diese ist derzeit am besten in Bezug auf die Rolle von IL-17A bei der Barrierefunktion im Darm erforscht. Die biologische Grundlage für die Bildung von IL-17 in der Wirbelsäule, die scheinbar unabhängig von IL-23 erfolgt, muss überprüft werden, und falls sie sich bestätigt, wirft dies die zentrale Frage auf, warum IL-17A so wichtig für die Immunbiologie der Wirbelsäule ist.

Diese Nichtlinearität zwischen IL-23 und IL-17 scheint es auch in der menschlichen Wirbelsäule zu geben, doch ist das Wissen diesbezüglich derzeit sehr rudimentär. Allerdings liegen erste Hinweise vor, nach denen der nachgeschaltete IL-17A-Signalweg in der axialen Biologie sowohl über IL-23 als auch über IL-23-unab-
106

Kompass Autoimmun 2021;3:100-107 DOI: $10.1159 / 000518361$ 
hängige Signalwege reguliert wird. Es sind weitere Untersuchungen beim Menschen erforderlich, die die Dosierung von IL-23 sowie eine sorgfältigen Bewertung von Krankheitssubtypen und objektiven Entzündungsmaßen, einschließlich der CRP-Werte und MRT-Manifestationen, beinhalten. Neu aufkommende Erkenntnisse zur Biologie der IL-23/17-Achse im menschlichen Skelett sprechen stark dafür, dass die gegenwärtig bestehende Komplexität einen verborgenen IL-23-Signalweg umfasst, und möglicherweise existierteineSpA-Untergruppemitaxialer Entzündung, bei der ein Antagonismus dieses Signalwegs noch therapeutisch nutzbar sein könnte.

\section{Beiträge der einzelnen Autoren}

C.B., A.W., K.S. und D.M. trugen alle zur wissenschaftlichen Diskussion, zum Schreiben und zur Erstellung der Abbildungen für die Arbeit bei. Alle Autoren waren an dem Artikel beteiligt und haben die eingereichte Fassung genehmigt.

\section{Disclosure Statement}

Die Autoren erklären, dass bei der Durchführung der Forschungsarbeit keine kommerziellen oder finanziellen Beziehungen bestanden, die als potenzieller Interessenkonflikt ausgelegt werden könnten.

\section{Lizenzangabe}

McGonagle D, Watad A, Sharif K, Bridgewood C. Why Inhibition of IL-23 Lacked Efficacy in Ankylosing Spondylitis. Front Immunol. $2021 \mathrm{Mar}$ 19;12:614255 (DOI: 10.3389/fimmu.2021.614255), ${ }^{\circ} 2021$ McGonagle, Watad, Sharif and Bridgewood (Übersetzung), lizensiert unter CC BY 4.0 (https://creativecommons.org/licenses/by/4.0/deed.de).

\section{Literatur}

Die Literatur ist unter www.karger.com/doi/10.1159/000518361 abrufbar. 